\title{
Kostenexplosions-Klagen gibt es schon lange
}

\author{
Willy Oggier \\ Dr. oec. HSG, Gesundheitsökonomische Beratungen AG, Küsnacht
}

1 Sommer Jürg H.

(23. September 1996). Kosten-«Explosion» im Gesundheitswesen Abflachung des Kostentrends ist nicht zu erwarten, in: Neue Zürcher Zeitung, 221
In der Ausgabe 11/2017 der Schweizerischen Ärztezeitung versucht der Soziologe Peter Streckeisen den Lesern glauben zu machen, dass Sparpolitik und Kostenexplosion seit der Nationalen Sparkonferenz im Gesundheitswesen vom 8. Mai 1982 ein Dauerthema sei. Diese Darstellung greift zu kurz.

\section{Leistungs-, nicht Kostenexplosion}

Wir haben es im Gesundheitswesen nicht mit einer Kosten-, sondern einer Leistungsexplosion zu tun. 1996, im Jahr der Einführung des eidgenössischen Krankenversicherungsgesetzes, formulierte der Basler Gesundheitsökonom Jürg H. Sommer folgende Gründe, warum der Kostenanstieg im Gesundheitswesen weitergehen wird ${ }^{1}$ :

- Rund 75 Prozent der sich krank oder unpässlich fühlenden Menschen konsultieren keinen Arzt. Es bestehen demnach auch bei wachsender Ärztedichte

Les plaintes au sujet de l'explosion des coûts ne datent pas d'hier

Dans le numéro 98 (11) du Bulletin des médecins suisses, Peter Streckeisen s'efforce de faire croire aux lecteurs que la politique d'économies et I'explosion des coûts sont un sujet de préoccupation permanent depuis la Conférence nationale d'économies en matière de santé publique du 8 mai 1982. Cette manière de présenter les choses est réductrice. Nous ne sommes pas confrontés à une explosion des coûts mais à une explosion des prestations dans la santé. La plus grande partie des hausses des coûts dans la santé est due aux possibilités croissantes d'intervention de la médecine. Cette évolution est encore favorisée par les incitations financières, qui sont orientées vers une expansion. L'expression "explosion des coûts» était par ailleurs utilisée longtemps auparavant dans le secteur de la santé. De la même manière qu'elle a pris de l'importance dans les débats publics, I'explosion (supposée) des coûts peut à nouveau être relativisée. Reste à savoir quels aspects domineront le débat à l'avenir.

Du point de vue de l'économie de la santé, la prééminence des soins de qualité dans une société vieillissante prend une importance particulière. II ne peut en effet y avoir d'économicité sans efficience des soins. Des efforts sont donc nécessaires, autant de la part des prestataires que des assureurs. noch genügend prinzipiell behandelbare gesundheitliche Störungen und Unpässlichkeiten in der Bevölkerung.

- In allen Industriestaaten wird davon ausgegangen, dass als Folge der demographischen Entwicklung die Anzahl der Betagten und Hochbetagten ansteigt. Empirische Untersuchungen zeigen allerdings, dass die Alterung der Bevölkerung nur einen geringen Teil des bis anhin eingetretenen Ausgabenwachstums im Gesundheitswesen erklären kann. Auf der

Empirische Untersuchungen zeigen, dass die Alterung der Bevölkerung nur einen geringen Teil des Ausgabenwachstums im Gesundheitswesen erklären kann.

Suche nach Marktlücken dürfte davon auszugehen sein, dass eine steigende Anzahl Ärztinnen und Ärzte den bis anhin eher vernachlässigten geriatrischen Bereich vermehrt entdeckt. Daher besteht die Gefahr, dass der Alterungsprozess von einer steigenden Zahl an Ärzten - ähnlich wie die Schwangerschaft und Geburt - zur Krankheit umfunktioniert wird.

- Wohlhabendere und besser gebildete Menschen nehmen in der Regel mehr Gesundheitsleistungen in Anspruch als ärmere und schlechter gebildete. Dies ist nicht weiter erstaunlich, weil die Medizin auch eine ausgeprägte Luxus- und Komfortkomponente enthält (z.B. umfassende Abklärung bei Bagatellen, Stationierung in den Privatabteilungen der Spitäler, Kuren, Massagen usw.). Mit zunehmendem Wohlstand ist daher zu vermuten, dass auch die Gesundheitsausgaben steigen.

- Der Grossteil der Kostensteigerungen im Gesundheitswesen ist auf die wachsenden Eingriffsmöglichkeiten der Medizin zurückzuführen, wobei die Fortschritte im Bereich der Diagnostik bedeutend rascher verliefen als jene bei den tatsächlichen Heilungsmöglichkeiten. Im Gegensatz zu technologischen Neuerungen in der Industrie wirken sich diese aber in der Medizin kostensteigernd aus, weil 
die neuen Apparaturen oft in Ergänzung zu den bisherigen verwendet werden und zusätzliches, speziell ausgebildetes Personal benötigen.

- Diese Entwicklung wird durch die auf Expansion ausgerichteten finanziellen Anreize noch gefördert. Einerseits übernimmt die Versicherung die entsprechenden Kosten für den Versicherten, anderseits

\section{Der Begriff der Kostenexplosion wurde im Gesundheitswesen schon viel früher verwendet.}

hat der Arzt keinerlei Anreize, bei seinen Entscheiden die Kostenseite einzubeziehen. Führt er beispielsweise aus Wirtschaftlichkeitsüberlegungen bestimmte Tests nicht durch, wird er dafür mit weniger Einnahmen und eventuell einem verärgerten Patienten bestraft.

\section{Kostenexplosions-Klagen vor 1982}

Hinzu kommt: Der Begriff der Kostenexplosion wurde im Gesundheitswesen schon viel früher verwendet. Dies machen folgende Zitate klar, die allesamt aus der Zeit vor dem behaupteten Beginn des Dauerthemas Kostenexplosion stammen: ${ }^{2}$

- Revision von 1964: «Fast alle Krankenkassen gewährten bereits unter dem alten KUVG auf freiwilliger Basis jene Leistungen, die ihnen heute vorgeschrieben werden! Hiezu gehören die Strahlentherapie, deren Kosten sich in schweren Fällen auf einige tausend Franken belaufen können,

2 Die Zitate sind der vom Konkordat der

Schweizerischen Krankenkassen (heute santesuisse) herausgegebenen Schrift von Jeanne Fell-Doriot, Die schweizerisch Krankenversicherung an einem Wendepunkt, Solothurn, 1967 und der dort zitierten Literatur entnommen.

Korrespondenz: Dr. oec. HSG Willy Oggier Boglerenstrasse $4 \mathrm{a}$ CH-8700 Küsnacht gesundheitsoekonom. willyoggier[at]bluewin.ch gen. Während aber der Bundesrat in seiner Botschaft zur Gesetzesvorlage annahm, dass diese Verbesserungen eine Erhöhung der Prämien von ungefähr 10 Prozent nach sich ziehen würde, stellt man heute fest, dass diese Erhöhung wesentlich stärker ins Gewicht fällt und bei gewissen Kassen 50 , ja sogar 70 Prozent erreicht.» (Fell-Doriot, 1967, S. 11)

- Das Spitalwesen ist überlebt: "Aus dem Gesagten zeigt sich, dass das heutige Spital überlebt ist. Einmal ist es dies in seiner finanziellen Konzeption. [...] Während sich die Löhne in diesem Zeitraum um das Drei- oder Dreieinhalbfache erhöht haben, sind die durchschnittlichen Spitalkosten pro Tag in den Bezirksspitälern des betreffenden Kantons um das Sechseinhalbfache und in einem Fall sogar um das Zwölffache angestiegen.» (Fell-Doriot, 1967, S. 29)

- Schlussfolgerungen: «Unsere Krankenversicherung kostet zuviel. 75 Prozent der Einnahmen der Kassen werden von den Versicherten selbst aufgebracht. Diese 75 Prozent stellen immer höhere Summen dar und übersteigen die finanziellen Möglichkeiten einer grossen Zahl von Versicherten.» (Fell-Doriot, 1967, S. 43)

Diese Zitate dürften zudem klar machen, dass der Begriff "Kostenexplosion" in seiner politischen Bedeutung im Lauf der Zeit ändern kann und vom Umfeld abhängt.

Genauso wie die (vermeintliche) Kostenexplosion in der öffentlichen Diskussion an Bedeutung gewonnen hat, kann sie daher auch wieder relativiert werden. Dies dürfte aus demographischen und demokratischen Gründen in älter werdenden Gesellschaften als gar nicht so abwegiges Szenario zu bezeichnen sein. Offen

\section{Wirtschaftlichkeit kann es ohne wirksame Versorgung gar nicht geben.}

bleibt die Frage, welche Aspekte in der Zukunft die Debatte dominieren werden.

Aus gesundheitsökonomischer Sicht kommt dabei dem Primat der guten Versorgung in einer älter werdenden Gesellschaft besondere Bedeutung zu. Denn Wirtschaftlichkeit kann es ohne wirksame Versorgung gar nicht geben. Leistungserbringer und Versicherer sind dabei gleichermassen gefordert. Soziologisches Lamentieren über die Ökonomisierung oder die Kostenexplosion bildet dabei keinen erfolgversprechenden Ansatz. Das Aufzeigen des Nutzens guter Versorgung dagegen eher schon.
Eine Replik von Peter Streckeisen folgt als Leserbrief in de nächsten Ausgabe der Schweizerischen Ärztezeitung. 